\title{
Long-term monitoring of donor xenogeneic testis tissue grafts and cell implants in recipient mice using ultrasound biomicroscopy
}

\begin{abstract}
Testis tissue xenografting and testis cell aggregate implantation from various donor species into recipient mice are novel models for the study and manipulation of testis formation and function in target species. Thus far, the analysis of such studies has been limited to surgical or postmortem retrieval of samples. Here we used ultrasound biomicroscopy (UBM) to monitor the development of neonatal porcine testis grafts and implants in host mice for $24 \mathrm{wk}$, and to correlate UBM and (immuno)histologic changes. This led to long-term visualization of gradual changes in volume, dimension and structure of grafts and implants; detection of a $4 \mathrm{wk}$ developmental gap between grafts and implants; and revelation of differences in implant development depending on the craniocaudal site of implantation on the back of host mice. Our data support the reliability and precision of UBM for longitudinal study of transplants, which eliminates the need for frequent surgical sampling.
\end{abstract}

Keyword: Male reproduction; Spermatogenesis; Testicular development; Testicular tissue xenografting; Testis cell implantation; Ultrasonography; Ultrasound biomicroscopy 